\title{
The Role of Cell Motility in Metastatic Cell Dominance Phenomenon: Analysis by a Mathematical Model
}

\author{
A.V. KOLOBOV ${ }^{a}$, A.A. POLEZHAEV ${ }^{\mathrm{a},{ }^{*}}$ and G.I. SOLYANIK ${ }^{\mathrm{b}}$ \\ ${ }^{\mathrm{a}}$ P.N. Lebedev Physical Institute, Leninsky prosp. 53. 117924 Moscow, Russia and ${ }^{\mathrm{b}}$ R.E. Kavetsky Institute of Experimental Pathology, \\ Oncology and Radiobiology, Vasilkovskaya 45, 252022 Kiev, Ukraine
}

(Received June 6, 1999; Revised November 5, 1999; In final form April 12, 2000)

\begin{abstract}
Metastasis is the outcome of several selective sequential steps where one of the first and necessary steps is the progressive overgrowth or dominance of a small number of metastatic cells in a tumour. In spite of numerous experimental investigations concerning the growth advantage of metastatic cells, the mechanisms resulting in their dominance are still unknown. Metastatic cell overgrowth occurs even if doubling time of the metastatic subpopulation is shorter than that of all others subpopulations in a heterogeneous tumour. In order to examine the hypothesis that under conditions of competition of cell subpopulations for common substrata cell motility of the slow-growing subpopulation can result in its dominance in a heterogeneous tumour, a mathematical model of heterogeneous tumour growth is suggested. The model describes two cell subpopulations which can grow with different rates and transform into the resting state depending on the concentration of the substrate consumed by both subpopulations. The slow-growing subpopulation is assumed to be motile. In numerical simulations it is shown that this subpopulation is able to overgrow the other one. The dominance phenomenon (resulting from random cell motion) depends on the motility coefficient in a threshold manner: in a heterogeneous tumour the slow-dividing motile subpopulation is able to overgrow its non-motile counterparts if its motility coefficient exceeds a certain threshold value. Computations demonstrate independence of the motile cells overgrowth from the initial tumour composition.
\end{abstract}

Keywords: tumour growth, cellular heterogeneity, motility, cell dominance

\section{INTRODUCTION}

One of the most important biological features of cancer, responsible for its lethality, is the ability of tumour cells to metastasize. Hence, identification of tumour cell characteristics involved in the metastatic process is of principle importance for understanding and treatment of malignant diseases.
It is known that tumour metastasis includes a complex succession of events whereby malignant cells spread from the primary tumour to colonise near and distant host tissues (Nicolson, 1987). An important feature of this process is that metastasis is the outcome of several selective sequential steps where one of the first and necessary steps is the progressive overgrowth or dominance of a small number of meta-

* Corresponding authors 
static cells in a tumour (Waghorne et al., 1988). The biological significance and implications of the metastatic cell dominance phenomenon for tumour biology has been discussed in detail (Miller et al., 1987 , Kerbel, 1990, Rak and Kerbel, 1993). In spite of numerous experimental investigations concerning the growth advantage of metastatic cells, the mechanisms resulting in their dominance are still unknown.

Metastatic process as a stage of a tumour progression is based on the main fundamental property of neoplasm - cellular heterogeneity (Bell et al., 1991, Symmans et al., 1995, Nicolson and Moustafa, 1998). In the framework of the heterogeneity concept, tumour progression is considered as a number of successive changes in the composition structure resulting from the host selection pressure: depletion of essential nutrients, chemotherapy and action of the host immunity. This selection can lead to dominance of the subpopulation with the growth advantage. It should be noted that such definition of the tumour progression is rather vague and it rises a number of questions. One of the main of them is the following: what inherent cellular characteristics give rise to the growth advantage of cellular population? The simplest and obvious answer is that it is the high rate of cell division. The experimental evidence of the overgrowth of the cell population with a shorter doubling time was obtained in investigations of the cellular composition of a heterogeneous tumour consisting of nonmetastatic cell subpopulations (Leith et al., 1987). However the analysis of other experimental data shows that dominance of metastatic cells is not directly associated with their proliferative characteristics (Staroselsky et al., 1990, Jouanneau et al., 1994). Moreover, metastatic cell overgrowth occurs even if doubling time of the metastatic subpopulation is longer than that of all others subpopulations in a heterogeneous tumour (Kerbel et al., 1987, Waghorne et al., 1988). What mechanisms ensure metastatic cell clonal dominance phenomenon in this case?

Some of the researchers consider cell-cell interactions to be responsible for the overgrowth of metastatic cells in a tumour (Theodorescu et al., 1991). From our point of view the attempts to explain the metastatic cell dominance phenomenon by the exist- ence of the specific cell-cell interactions, in the framework of which, for example, non-metastatic cells produce growth factors which preferentially stimulate growth of metastatic ones, shift the solution of the problem towards the search of specific proteins and/or receptors resulting in such interactions. Other researchers suggest that the ability of metastatically-competent cells to dominate is due to high locomotory capacity of these cells compared to their non-metastatic counterparts (Solyanik et al., 1995, Solyanik, 1998). The important role of cellular motility in the metastatic cascade is well known (Weiss, 1990, Zanker, 1997, Zaizen et al., 1998, Uchida et al., 1999). But it seems doubtful that for the sufficient and spatially homogeneous concentration of nutrients the motility of cells may result in their overgrowth in a tumour. Meanwhile under the limited and heterogeneous environmental conditions resulting from the competition of cells for nutrients (the simplest form of cell-cell interactions) the motile cells can leave the zone with low substrate level and invade the tumour and surrounding tissues where better nutrient conditions provide the basis for their proliferation and advantage to overgrow non-motile cell subpopulations.

The purpose of the present investigation is to examine the hypothesis that under conditions of competition of cell subpopulations for common substrata cell motility of a subpopulation can give rise to its growth advantage over other non-motile cells even if the motile subpopulation doubling time is longer than that of other cell subpopulations in a heterogeneous tumour. This hypothesis is tested by a mathematical model for the growth dynamics of a heterogeneous tumour suggested by us.

Commonly the description of cell population growth is based on the equations of the reaction-diffusion type with logistic (likes Verhulst law) cell population birth-death processes. It should be noted that the form of the source term in these equations reflects the fundamental empirical observation that the increase in cancer cell number is accompanied by the slowing down of the tumour growth rate (Burton, 1966, Byrne and Chaplain, 1995, Greenspan, 1972, Gatenby, 1996, Perumpanani et al., 1996). But in 
some cases, and especially for tumour growth, this approach has one essential drawback: the system behaviour is determined by local kinetics and own cell motility only that may result in overestimation of some model parameters such as motility coefficients. For example, Traqui (1995) from fitting of the reaction-diffusion model to experimental data estimated cell motility coefficient to be of the order of $10^{-7}$ $\mathrm{cm}^{2} / \mathrm{sec}$ that is the same as for diffusion coefficients of large macromolecules, which seems to be doubtful. Moreover in the limits of the traditional description growth of non-motile tumour population appears to be impossible. Thus, the development of the tumour population with the total cell density limited due to the effects of overcrowding and tight packing should depend on other factors which are not described in the limits of traditional reaction-diffusion systems. As is shown below it is convenient to introduce an "effective" convection process due to which the spatial cell distribution changes enabling further growth of the population.

It should be noted that our model doesn't take into account cell adhesion although a correlation between adhesion of cells to the components of extracellular matrix and invasion has been shown (Volk et al., 1984, Kornberg, 1998). However, this correlation is supposed to be due to a co-ordinated interplay between cytokine-mediated responses and extracellular matrix-directed regulation of cellular adhesion and motility (Silletti et al., 1998, Herrera-Gayol and Jothy, 1999). These cell functions are coupled through unknown mechanisms and thus it is hard to consider correctly both cell adhesion and motility in the framework of the same mathematical model.

It is known that cancer cell motility may be spontaneous or may be stimulated by different cytokines or by the components of the extracellular matrix. The cell migrating response depends on the concentration of attractants and includes both random (chemokinesis) and directed components of motion (chemo- and haptotaxis). In the present study we consider only random motility of cells because, on the one hand, it is the simplest form of cell motion, which takes place in absence of external specific biological factors, and on the other hand, though correlation between in vitro motility and in vivo invasiveness ${ }^{*}$ has been shown in numerous studies (Takaoka et al., 1998, Bourguignon et al., 1998, Siegel et al., 1998), there are some observations indicating that the increased chemotactic response of cancer cells is an evidence of a reduced metastatic potential (Geng et al. 1998).

\section{MATHEMATICAL MODEL}

The model which we propose for tumour growth is based on the following assumptions:

- A tumour grows as a spherical colony of malignant cells. Surrounding medium does not hinder tumour growth and malignant cell motion. This assumption is justified by the high activity of proteolytic cascade in metastatically competent cells required for degradation of neighbouring tissues (Ginestra et al., 1998).

- A tumour consists of two subpopulations, denoted by $a$ and $c$, which differ in cell properties and thus in their growth kinetics.

- Each subpopulation includes pools of proliferating $-a_{1}, c_{1}$ and resting $-a_{2}, c_{2}$ cells.

- Proliferating cells divide with the constant rate and under certain conditions can transform into the resting state. The rate of this transition depends on the intracellular level of crucial metabolites.

- We neglect the reverse transition from the resting state into the proliferating one.

- Interaction between subpopulations is manifested in the competition for the common substrate. Interaction between tumour cells and host immunity system is not taken into account.

- We assume that proliferating cells move randomly with the motility coefficients $D_{a}$ and $D_{c}$ respec-

\footnotetext{
* Invasion is considered as an early extratumour step in the metastatic process (in contrast to the metastatic dominance phenomenon which is an intratumour event in the metastatic cascade). The correlation between the invasiveness and metastatic ability of cancer cells has been demonstrated in a number of investigations (Takaoka et al., 1998, Uemura et al., 1998). In the present research we use the term "invasiveness" as a synonym of "metastatic ability" though we are aware of incompetence of such unification of these terms for other cases.
} 
tively, which are the same for the intra- and extratumour regions.

- Spatial substrate distribution is determined by balance between the substrate diffusion from the outside of the tumour region and its consumption by cycling tumour cells.

The model which is based on the above assumptions consists of five equations: four equations for the respective cell densities and an equation for the substrate concentration. Growth kinetics of tumour cell subpopulations is described by the following set of differential equations:

$$
\begin{aligned}
& \dot{a}_{1}=B_{a} \cdot a_{1}-P_{a}(S) \cdot a_{1}-\nabla . \mathrm{J}_{a 1}, \\
& \dot{a}_{2}=P_{a}(S) \cdot a_{1}-\nabla . \mathrm{J}_{a 2}, \\
& \dot{c}_{1}=B_{c} \cdot c_{1}-P_{c}(S) \cdot c_{1}-\nabla . \mathrm{J}_{c 1}, \\
& \dot{c}_{2}=P_{c}(S) \cdot c_{1}-\nabla . \mathrm{J}_{c 2},
\end{aligned}
$$

where the terms $B_{a}$ and $B_{c}$ describe the subpopulation growth rates, $P_{a}(S)$ and $P_{c}(S)$ represent transition functions from the cycling to the resting state depending on the substrate level and $\mathbf{J}$ are fluxes of the corresponding cells consisting of two components: the first of them is determined by the active random cell motion described by the Fick's law $-D_{a} \nabla a_{1}$ (in the present study we assume that only the cells $a_{1}$ are able to move) and the second is caused by the convection motion and is equal to the product of the corresponding cell density on the convective velocity.

Solving the equations (1) we take into account that unlike chemical molecules cells have considerable volume. It means that there is an essential limitation on the total cell density:

$$
\sum a_{i} \cdot V_{a i}+\sum c_{i} \cdot V_{c i} \leq 1
$$

where $V_{i}$ - are average volumes of the corresponding cell types. Obviously, cells of different subpopulations and in different states (proliferating or resting) differ in their volumes but further for simplicity we neglect these differences and assume cells to be of the same volume $V_{a}=V_{c}=A^{-1}$ as it does not effect qualitatively the results of modelling.

After some manipulation, the details of which are given in the Appendix, the set (1) with the account of the condition (2) in the spherically symmetrical case is transformed to the following form:

$$
\begin{aligned}
& \dot{a}_{1}= B_{a} \cdot a_{1}-P_{a}(S) \cdot a_{1} \\
&+\frac{1}{r^{2}} \frac{\partial}{\partial r}\left(D r ^ { 2 } \left(1-\frac{a_{1}}{A} \theta\left(\sum_{i}\left(a_{i}+c_{i}\right)\right.\right.\right. \\
&\left.-A)) \frac{\partial a_{1}}{\partial r}\right)-\theta\left(\sum_{i}\left(a_{i}+c_{i}\right)-A\right) \frac{1}{A} \\
& \times\left(\left(B_{a} a_{1}+B_{c} c_{1}\right) a_{1}\right. \\
&\left.+\frac{\partial a_{1}}{\partial r} \cdot \frac{1}{r^{2}} \int_{0}^{r} \rho^{2}\left(B_{a} a_{1}+B_{c} c_{1}\right) d \rho\right) \\
& \dot{a}_{2}= P_{a}(S) \cdot a_{1}-\theta\left(\sum_{i}\left(a_{i}+c_{i}\right)-A\right) \frac{1}{A} \\
& \times\left(\left(B_{a} a_{1}+B_{c} c_{1}\right) a_{2}+\frac{1}{r^{2}} \frac{\partial}{\partial r}\left(D r^{2} a_{1} \frac{\partial a_{2}}{\partial r}\right)\right. \\
&\left.+\frac{\partial a_{2}}{\partial r} \cdot \frac{1}{r^{2}} \int_{0}^{r} \rho^{2}\left(B_{a} a_{1}+B_{c} c_{1}\right) d \rho\right), \\
&\left.+\frac{\partial c_{2}}{\partial r} \cdot \frac{1}{r^{2}} \int_{0}^{r} \rho^{2}\left(B_{a} a_{1}+B_{c} c_{1}\right) d \rho\right), \\
& \dot{c}_{2}= P_{c}(S) \cdot c_{1}-\theta\left(\sum_{c}\left(a_{i}+c_{i}\right)-A\right) \frac{1}{A} \\
&\left.+\frac{\partial c_{1}}{\partial r} \cdot \frac{1}{r^{2}} \int_{0}^{r} \rho^{2}\left(B_{a} a_{a} a_{1}+B_{c} c_{1}\right) d \rho\right), \\
& B_{1}-\theta\left(B_{i}\left(a_{i}+c_{i}\right)-A\right) \\
&\left.\dot{c}_{1}+B_{c} c_{1}\right) c_{1}+\frac{1}{r^{2}} \frac{\partial}{\partial r}\left(D r^{2} a_{1} \frac{\partial c_{1}}{\partial r}\right)
\end{aligned}
$$

where $\theta(x)$ equals unity for $x \geq 0$ or else is zero. The presence of this function in the equations reflects the fact that outside the region of tightly packed tumour cells their motion is not hindered by the host tissue cells and thus the set (3) is reduced there to the conventional reaction-diffusion equations.

Evidently the forms of $P_{a}(S)$ and $P_{c}(S)$. which represent transition functions, significantly influence the tumour growth kinetics. It is clear that for the high substrate concentrations, which are sufficient to keep 
up the mitotic activity of the cells, the transition functions should tend to zero: $P_{i}(\infty)=0$, while for low level of concentrations their values should exceed the rates of cell division: $P_{i}(0)>B_{i}$. Thus we take the transition functions in the following form:

$$
P_{i}(S)=B_{i} \cdot K_{i} \cdot \exp \left(-S / S_{i 0}\right),
$$

where $K_{i}$ is the ratio of the maximal rate of cell transition to the resting state and the cell division rate $\left(K_{i}>\right.$ 1) and $S_{i 0}$ is the critical value of substrate below which metabolite depletion begins. We assume that the parameters in the expression (4) are the same for both subpopulations:

$$
K_{a}=K_{c}=K, \quad S_{a 0}=S_{c 0}=S_{0}
$$

In general this assumption may not be true but we neglect the possible difference in the corresponding parameter values as it does not change qualitatively the behaviour of the system.

The substrate distribution is described by the following equation:

$$
\dot{S}=Q(S)+D_{s} \Delta S,
$$

where $D_{s}$ is the substrate diffusion coefficient and $Q(S)$ is the rate of substrate consumption by cells of both subpopulations. In the present paper we consider oxygen as a crucial nutrient and this assumption is supported by numerous experimental data (Sutherland 1998). If necessary the influences of other possible nutrients (glucose, for example) on the growth kinetics of a heterogeneous tumour can be also included into the model.

For numerous cell lines it has been shown that oxygen consumption rate per cell is a function of oxygen concentration with the Michaelis-Menten type kinetics. Thus we assume the following expression for the function $Q(S)$ :

$$
Q(S)=-q_{s} \cdot \frac{S}{S+S^{*}}\left(a_{1}+k_{e f f} \cdot c_{1}\right)
$$

We neglect oxygen consumption by quiescent cells of both subpopulations as it is clear that for proliferation a higher rate of substrate consumption is necessary compared to the resting state.
The set (3) with the account of equations (5) and (6) is solved in a circular symmetrical domain of the radius $R$ with the following boundary and initial conditions:

$$
\begin{aligned}
\left(a_{1}\right)_{r}^{\prime}(0, t) & =\left(a_{1}\right)_{r}^{\prime}(R, t)=\left(c_{1}\right)_{r}^{\prime}(0, t)=\left(c_{1}\right)_{r}^{\prime}(R, t) \\
& =S_{r}^{\prime}(0, t)=0 ; \\
S(R, t) & =S_{\text {ext }} ; \\
a_{2}(r, 0) & =c_{2}(r, 0)=0, \\
S(r, 0) & =S_{\text {ext }}, \quad 0 \leq r \leq R ; \\
a_{1}(r, 0) & =\left\{\begin{array}{c}
A \cdot a^{*}, 0 \leq r \leq R_{0} \\
0, R_{0}<r \leq R
\end{array} ;\right. \\
c_{1}(r, 0) & =\left\{\begin{array}{c}
A\left(1-a^{*}\right), 0 \leq r \leq R_{0} \\
0, R_{0}<r \leq R
\end{array} .\right.
\end{aligned}
$$

In our simulations we assume that a homogeneous mixture of $a_{1}$ and $c_{1}$ cells initially occupy a small volume of the radius $R_{0} \ll R$ near the origin.

\section{RESULTS}

The model suggested was applied for simulation of changes in densities of cell subpopulations and oxygen concentration in space and time. Values of constants and parameters used in computations are given in Table I. It should be noted that in all model simulations the division rate of the subpopulation ' $a$ ' was considered to be twice less than that of the subpopulation ' $\mathrm{c}$ ': $2 \cdot B_{a}=B_{c}$, therefore the subpopulation ' $\mathrm{a}$ ' is further called a slow-dividing subpopulation and the subpopulation ' $\mathrm{c}$ ' - a fast-dividing subpopulation.

The results obtained show that composition dynamics of the model significantly depends on the value of cell diffusion $D_{a}$, demonstrating the possibility of the motile subpopulation to dominate in the heterogeneous tumour when diffusion rate $D_{a}$ exceeds some threshold value (Figure 1). The slow-dividing motile subpopulation with the motility coefficient which is under the threshold value has no chance to overgrow its non-motile counterparts even if the motile subpopulation constitutes the majority of the cells at the beginning of the tumour growth (Figure 2). 

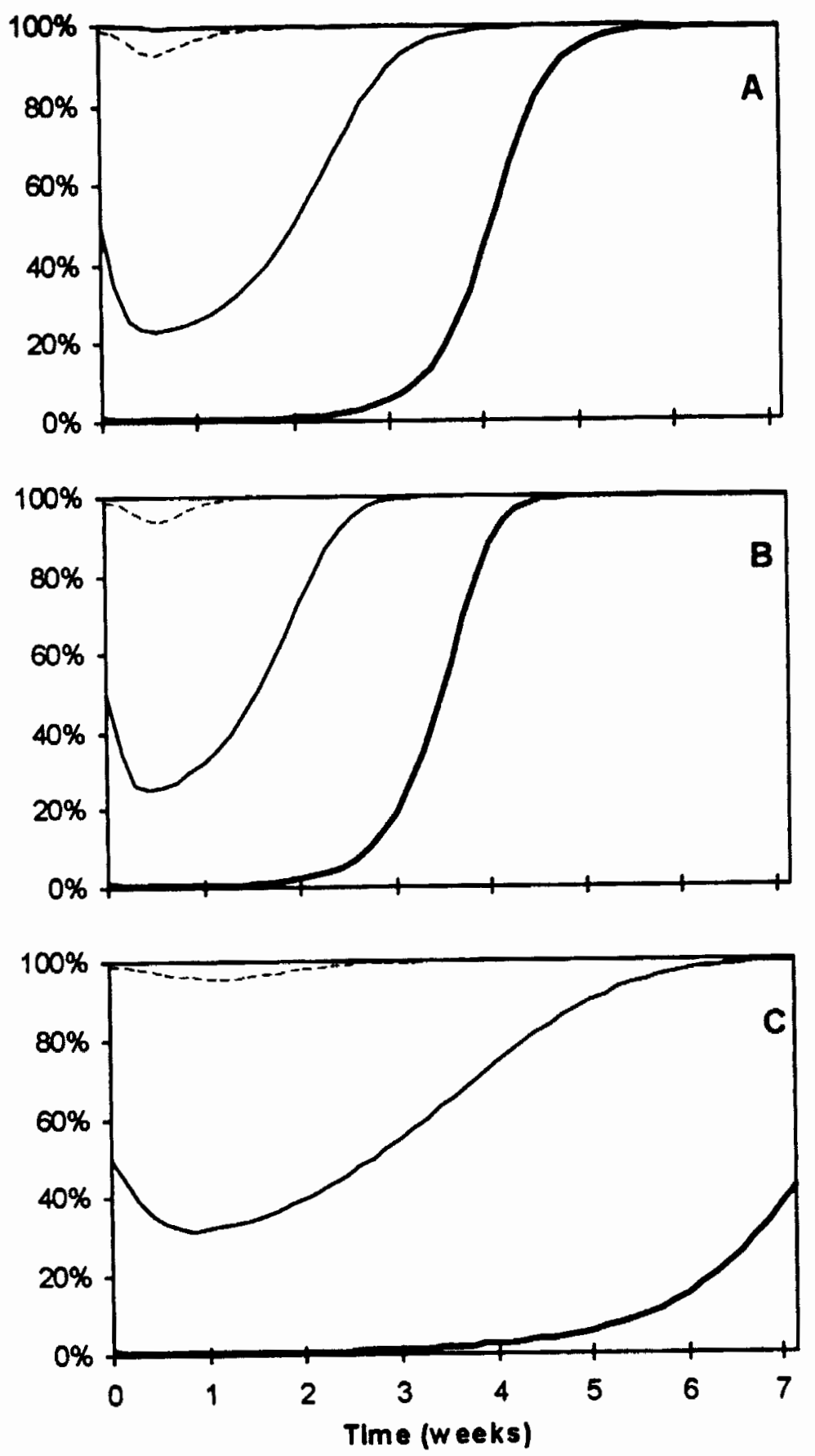

FIGURE 1 Time-dependent cellular composition of a tumour (only proliferating cell are taken into account) obtained numerically. Relative cell density of the subpopulation $a_{1}$ is shown. Initial compositions $a$ are $1 \%$-thick solid line; $50 \%$ - thin solid line; $99 \%$ - dotted line. Simulations are made for the cell motility coefficient $D_{a}=10^{-9} \mathrm{~cm}^{2} / \mathrm{sec}$ and for different values of $K$ and $S_{0}:$ A) $K=1.2 ; S_{0}=0.2 \cdot S_{e x t}$, B) $K=1.5$; $S_{0}=0.2 \cdot S_{\text {ext }}$, C) $K=1.5 ; S_{0}=0.8 \cdot S_{\text {ext }}$ 

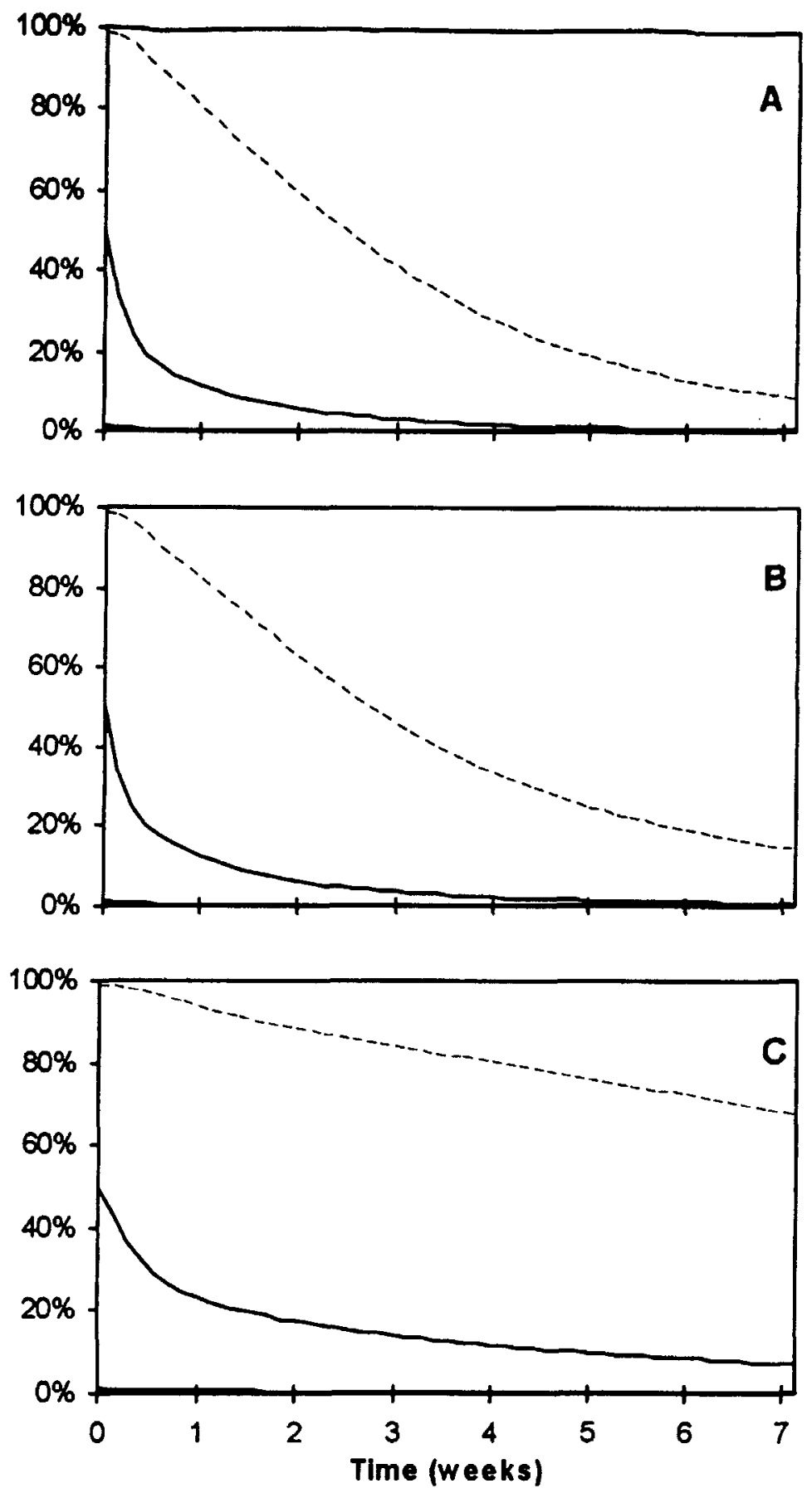

FIGURE 2 Time-dependent cellular composition of a tumour (only proliferating cell are taken into account) obtained numerically. Relative cell density of the subpopulation $a_{1}$ is shown. Initial compositions $a^{*}$ are $1 \%$-thick solid line; $50 \%$ - thin solid line; $99 \%$ - dotted line. Simulations are made for the cell motility coefficient $D_{a}=10^{-10} \mathrm{~cm}^{2} / \mathrm{sec}$ and for different values of and $S_{0}:$ A) $\left.\mathrm{K}=1.2 ; S_{0}=0.2 \cdot S_{E x t}, \mathrm{~B}\right) \mathrm{K}=1.5$; $S_{0}=0.2 \cdot S_{e x t}$, C) $K=1.5 ; S_{0}=0.8 \cdot S_{\text {ext }}$ 
TABLE I Model constants and parameters

\begin{tabular}{|c|c|c|}
\hline \multicolumn{3}{|c|}{ Constants } \\
\hline$q_{s}=1.7 \cdot 10^{-17}$ & moles/(cell $\cdot$ sec $)$ & Constants taken \\
\hline$D_{s}=3.0 \cdot 10^{-5}$ & $\mathrm{~cm}^{2} / \mathrm{sec}$ & from literature \\
\hline$S^{*}=4.2 \cdot 10^{-6}$ & moles & (Casciari et al., 1992) \\
\hline$S_{e x t}=2.8 \cdot 10^{-4}$ & moles & \\
\hline$B_{a}=0.03$ & $h^{-1}$ & Values assumed for \\
\hline$B_{c}=0.06$ & $h^{-1}$ & tumor cells \\
\hline$V_{a}=V_{c}=10^{-9}$ & $\mathrm{~cm}^{3}$ & \\
\hline$R_{\max }=1$ & $\mathrm{~cm}$ & \\
\hline$R_{0}=2 \cdot 10^{-2}$ & $\mathrm{~cm}$ & \\
\hline \multicolumn{3}{|l|}{$k_{e f f}=2$} \\
\hline \multicolumn{3}{|c|}{ Parameters used in simulations } \\
\hline$D_{a}=10^{-10} ; 10^{-9}$ & $\mathrm{~cm}^{2} / \mathrm{sec}$ & \\
\hline$S_{0}=5.6 \cdot 10^{-5} ; 22 \cdot 10^{-4}$ & moles & \\
\hline$K=1.2 ; 1.5$ & & \\
\hline
\end{tabular}

The model simulations show that initial composition cannot qualitatively change the result of tumour development (Figure 1,2) contrary to the results obtained for a spatially non-distributed system (Solyanik et al., 1995). Moreover in the case of the domination of slow-dividing subpopulation conversion to the monoclonal tumour occurs faster than in the opposite case and the initial composition less influence the time of the subpopulation overgrowth.

The sensitivity to the critical oxygen level $S_{0}$ is higher than to the changes in the maximum rate of cell transition to the resting state $K$ but cannot fundamentally modify cellular composition dynamics of the heterogeneous tumour (Figure 1,2). The changes of the parameter values $K$ (Figure 1A, 1B) and $S_{0}$ (Figure 1B, 1C) influence the time of the motile subpopulation overgrowth and may slightly change the threshold value of $D_{a}$.

Spatial cell distribution for one particular set of parameters, where $D_{a}$ exceeds the threshold value, is given in Figure 3. One can see that the process of slow-dividing subpopulation overgrowth can be divided into three stages: first, motile cells run away from the primary looking for the better environmental conditions (10th day of growth); second, under sufficient level of nutrient motile cells begin active prolif- eration (20th day of growth); third, immobile cells become extinct due to shortage of the substrate, while motile cells continue proliferation in normal environmental conditions( 30 th day of growth).

On the contrary, when the cell motility coefficient does not exceed the threshold value the slow-dividing subpopulation can't run away from the primary tumour and thus can't wipe out the counterpart (Figure 4).

Thus, in the framework of our model, the possibility of slow- dividing but motile subpopulation overgrowth in a heterogeneous tumour is shown. It is worth mentioning that model simulations demonstrate good qualitative agreement with the experimental data obtained by Theodorescu et al. (1991).

\section{DISCUSSION}

It is known that cell motility is a prerequisite for tumour cell invasion and is thought to be a key property in a metastatic cascade. The direct correlation between motility of cancer cells in vitro and their metastatic activity in vivo was shown in many experimental investigations (Volk et al., 1984, Hynes and Lander, 1992, Ziober et al., 1999). These observations underlie the main idea of our investigation about the important role of cell motility in metastatic cell dominance phenomenon taking into account that the later is necessary for distant metastatic spread. The results, presented above, confirm this hypothesis showing that the migrating capacity of cells (as an inherent cellular characteristic) can result in their growth advantage in a heterogeneous tumour. Three separate, but bound up with each other, basic stages were shown to be involved in dominance of the motile subpopulation over the fast-growing one. The first is the locomotion of motile cancer cells through host tissues; the second is related to the active proliferation of motile cells in advantageous environmental conditions; the third involves death of non-motile fast-dividing cells due to the lack of nutrients. It should be noted that the first and the second stages are known to underlie invasion. In the case of human invasive cutaneous melanoma it was shown that invasion toward the cutaneous tissues 

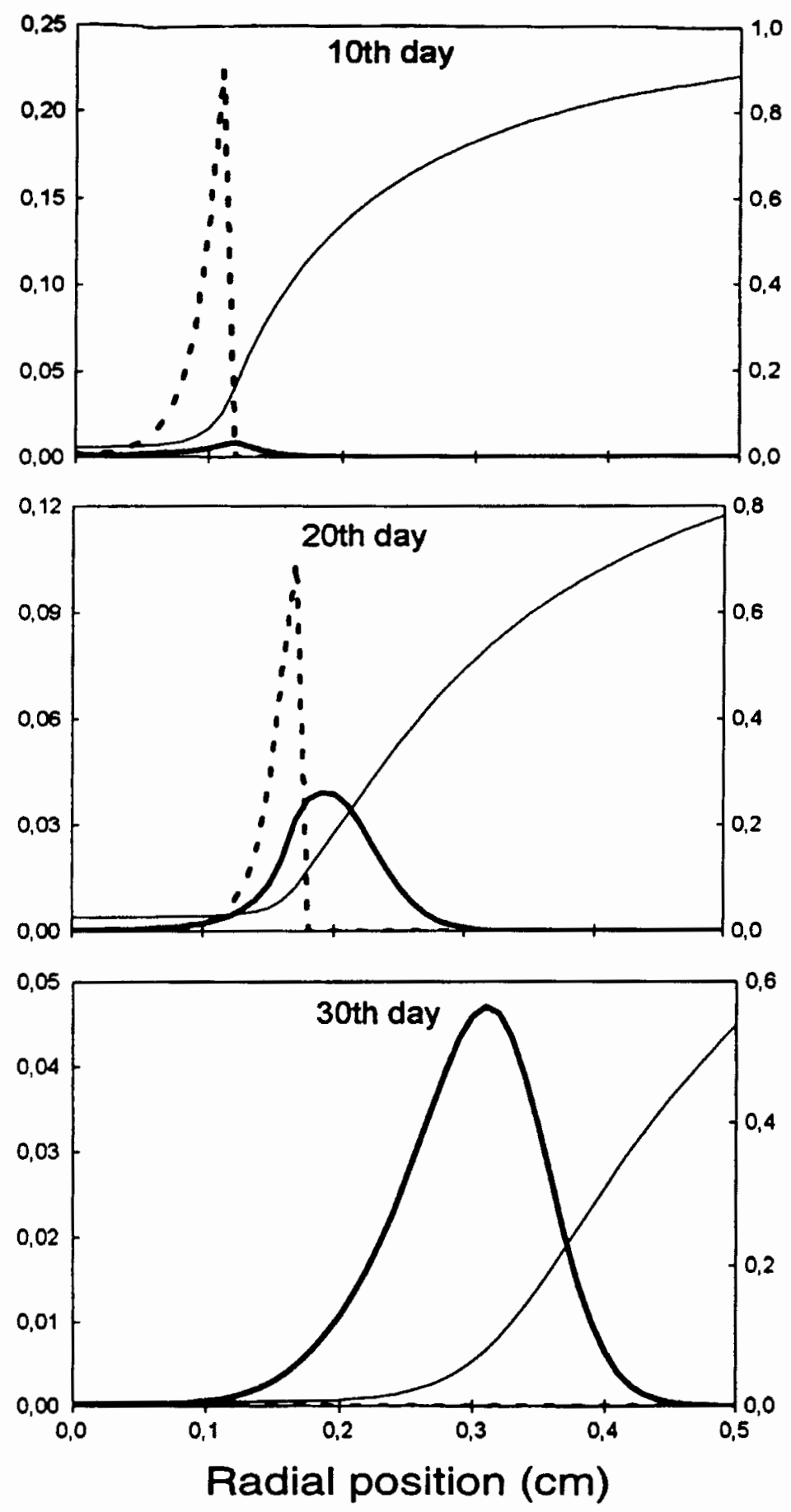

FIGURE 3 Numerical simulation for spatial cell and substrate distributions in a growing tumour; all parameter values are the same as in Figure 1B. Initial composition $a^{*}$ equals $10 \%$. Relative cell densities of the subpopulations $a_{1}$ (thick solid) and $c 1$ (thick dotted) are shown on the left scale. (Maximum cell density $A$ is taken equal to unity.) Substrate concentration $S$ (thin solid line) is given on the right scale in relative values. (External oxygen concentration $S_{e x t}$ is taken equal to unity.) 

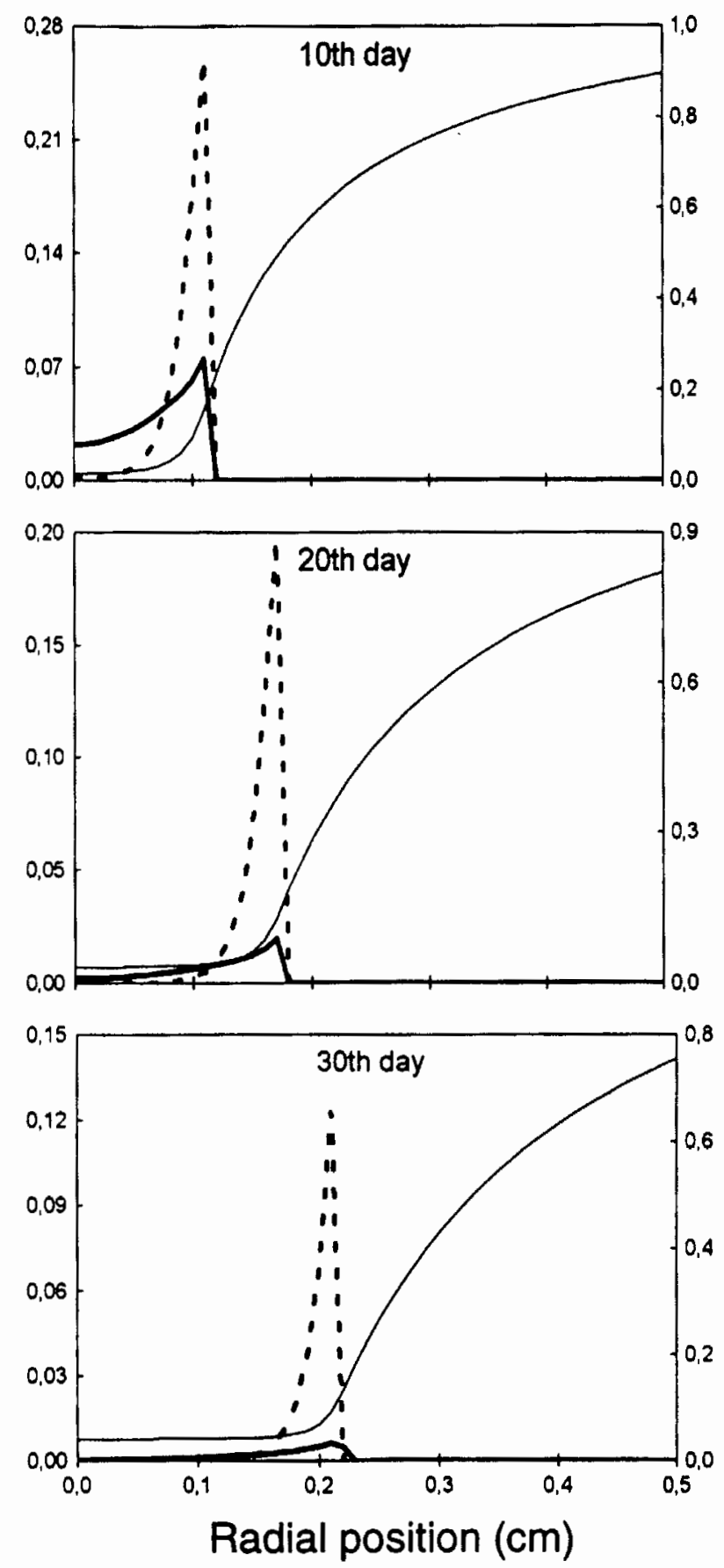

FIGURE 4 Numerical simulation for spatial cell and substrate distributions in a growing tumour; all parameter values are the same as in Figure 2B. Initial composition $a^{*}$ equals $90 \%$. Relative cell densities of the subpopulations $a_{1}$ (thick solid) and $c_{1}$ (thick dotted) are shown on the left scale. (Maximum cell density $A$ is taken equal to unity.) Substrate concentration $S$ (thin solid line) is given on the right scale in relative values. (External oxygen concentration $S_{e x t}$ is taken equal to unity.) 
occurs by repetitive cycles of active locomotion of melanoma cells followed by cessation of movement and proliferation (Suh and Weiss, 1984, Weiss, 1990). Thus our investigation demonstrate that invasion and growth dominance of the metastatic cancer cells are realised through the same mechanisms, based on the same inherent cellular characteristics of cancer cells, and appear to be two manifestations of one process occupation of space, both intratumour and extratumour, by the metastatically competent cells.

The well-known peculiarity of invasion is the crucial role of time in manifestation of this phenomenon. A number of mechanisms are considered to account for the long delay in the transition from in situ to invasive carcinomas (Weiss, 1990). One of the possible mechanisms of this delay results from gradual emergence of a motile subpopulation of cancer cells in the process of tumour progression. Our results demonstrate that the dominance phenomenon as well as invasion (resulting from the random cell motion) depends on the motility coefficient in a threshold manner. It means that the appearance of a motile subpopulation does not guarantee this subpopulation to be invasive. To run away from the primary tumour and thus to overgrow all other subpopulations the cells of the motile subpopulation should have diffusion rate exceeding a certain threshold value. As was shown above the value of this "invasive" diffusion rate considerably depends on the proliferative characteristics of both subpopulations (the rates of cell division, the rates of cell transition into the resting state, the sensitivity to the oxygen depletion) and ranges from $10^{-10} \mathrm{~cm}^{2} / \mathrm{sec}$ to $10^{-9} \mathrm{~cm}^{2} / \mathrm{sec}$. Thus the suggested model determines the inherent cellular characteristics resulting in the tumour invasion that provides the growth preference of metastatically competent cells in a primary tumour and gives the possibility to make some quantitative estimates for these cellular characteristics.

It should be noted that the threshold relationship between the cell motility and cell dominance phenomenon is determined to a large extent by the assumption made that surrounding medium does not hinder tumour growth and cancer cell motion. In the framework of other possible assumption when tumour growth is considered to be accompanied by ousting of host tissue and there is no antagonistic interaction between tumour and host cells the slow-dividing population with any motility coefficient should always run away from the primary tumour and so it should always overgrow its non-motile counterpart. In this case dominance phenomenon (resulting from the random cell motion) should not depend on the diffusion coefficient in a threshold manner.

The model demonstrates that the final overgrowth of motile cells does not depend on the initial cellular composition of the tumour. Although this result has its experimental confirmation, the data obtained by Staroselsky et al. (1990) seem to contradict it. These investigators didn't observe dominance of metastatic cells if the portion of these cells in the initial composition of the tumour is less than $5 \%$. However from our point of view, this difference between model predictions and experimental observations may follow from stochastic nature of cell survival after the injection of a small number of metastatic cells in animals. This stochasticity appears as a result of cell-host interactions determining the existence of the minimum number of cancer cells which is necessary for successful tumour inoculation. Apparently this effect cannot be described by our model.

As was mentioned above cell motility includes both random and directed components of motion. Despite numerous studies the question of relative contributions of the chemotactic and chemokinetic responses in tumour progression is still open. In the present paper we considered the simplest form of cell motility - chemokinesis with substrate-independent motility coefficient, and showed that just this type of motion is sufficient to ensure overgrowth of slow-dividing metastatic cells in a heterogeneous tumour. The question which is to be clarified is the following: what chemokinesis and chemo- and haptotaxis can contribute to the variety of cell behavioural patterns in the growth dominance of metastatic cells. Migrating response of cancer cells to chemoattractive ingredients is evidently important for organ-specific metastasis (Ito et al., 1998), however the problem is whether it can add anything qualitatively new to the overgrowth of metastatic cells in an early tumour. The 
answer to this question requires additional investigations, both experimental and theoretical.

The work was financially supported by the Russian Foundation of Basic Research.

\section{References}

[1] Bell, C., Frost, P. and Kerbel, R.S. (1991). Cytogenetic heterogeneity of genetically marked and metastatically competent "dominant" tumor cell clones. Cancer Genetics and Cytogenetics, 54(2), 153-161.

[2] Bourguignon, L.Y., Gunja-Smith, Z., Iida, N., Zhu, H.B., Young, L.J., Muller, W.J. and Cardiff, R.D. (1998). CD44v(3,8-10) is involved in cytoskeleton-mediated tumor cell migration and matrix metalloproteinase (MMP-9) association in metastatic breast cancer cells. Journal of Cellular Physiology, 176, 206-215.

[3] Burton, A.C. (1966). Rate of growth of solid tumours as a problem of diffusion. Growth, 30, 159-176.

[4] Byrne, H.M. and Chaplain, M.A.J. (1995). Growth of non-necrotic tumors in the presence and absence of inhibitors. Mathematical Biosciences, 130, 151-181.

[5] Casciari, J.J., Sotirchos, S.V. and Sutherland, R.M. (1992). Mathematical modelling of microenvironment and growth in emt6.ro multicellular tumor spheroids. Cell Proliferation, 25, $1-22$.

[6] Gatenby, R.A. (1996). A reaction-diffusion model of cancer invasion. Cancer Research, 56(24), 5745-5753.

[7] Geng, L., Ali, S.A., Marshall, J.F., Mackay, C.L., Hart, I.R., Delcommence, M., Streuli, C.H. and Rees, R.C. (1998). Fibronectin is chemotactic for CT 26 colon carcinoma cells: sub-lines selected for increased chemotaxos to fibronectin display decreased tumorigenicity and lung colonization. Clinical and Experimental Metastasis, 16, 683-691.

[8] Ginestra, A., La Placa, M.D., Saladino, F., Cassara, D., Nagase, H. and Vittorelli, M.L. (1998). The amount and proteolytic content of vesicles shed by human cancer cell lines correlates with their in vitro invasiveness. Anticancer Research, 18(5A), 3433-3437.

[9] Greenspan, H.P. (1972). Models for the growth of a solid tumour by diffusion. Studies in Applied Mathematics, 51, 317-340.

[10] Gusev, A. and Polezhaev, A. (1997). Modeling of a cell population evolution for the case existence of maximal possible total cell density. Kratkie soobscheniya po fizike FIAN, 11$12,85-90$

[11] Herrera-Gayol, A. and Jothy, S. (1999). CD44 modulates Hs578T human breast cancer cell adhesion, migration and invasiveness. Experimental and Molecular Pathology, 66, 99-108.

[12] Hynes, R.O. and Lander, A.D. (1992) Contact and adhesive specifities in the associations migrations and targeting of cells and axons. Cell, 68, 303-322.

[13] Ito, H., Miyazaki. M., Nishimura, F. and Nakajima, N. (1998). Haptotactic migration of pancreatic cancer cells induced by bioactive components in bovine liver extract. Journal of Surgical Oncology, 68, 153-158.

[14] Jouanneau, J., Moens, G., Boirgeois, Y., Poupon, M.F. and Thiery, J.P. (1994). A minority of carcinoma cells producting acidic fibroblast growth factors induces a community effect for tumour progression. Proceedings of the National Academy of Sciences USA, 91, 286-290.
[15] Kerbel, R.S., Waghorne, C., Man, M.S., Elliott, B.E. and Breitman, M.L. (1987). Alteration of the tumorigenic and metastatic properties of neoplastic cells is associated with the process of calcium phosphatemediated DNA transfection Proceedings of the National Academy of Sciences USA, 84 $1263-1267$.

[16] Kerbel, R.S. (1990). Growth dominance of the metastatic cancer cells: cellular and molecular aspects. Advances in Cancer Research, 55, 87-131.

[17] Kornberg, L.G. (1998). Focal adhesion kinase and its potential involvement in tumor invasion and metastasis. Head Neck, 20, 745-752.

[18] Leith, J.T., Michelson, S., Faulkner, L.E. and Bliven, S.F. (1987). Growth properties of artificial heterogeneous human colon tumors. Cancer Research, 47, 1045-1051.

[19] Miller, B., Miller, R. and Hepnner, G. (1987). Analysis of tumor cell composition in tumors of paired mixtures of mammary cell lines. British Journal of Cancer, 56, 561-569.

[20] Nicolson, G.L. (1987). Tumour cell instability, diversification, and progression to the metastatic phenotype: from oncogene to oncofetal expression. Cancer Research, 47, 14731487.

[21] Nicolson, G.L. and Moustafa, A.S. (1998). Metastasis-associated genes and metastatic tumor progression. In vivo, 12(6), 579-588.

[22] Perumpanani, A.J., Sherratt, J.A., Norbury, J. and Byrne, H.M. (1996). Biological inferences from a mathematical model for malignant invasion. Invasion Metastasis, 16, 209221.

[23] Rak, J.W. and Kerbel, R.S. (1993). Growth advantage ("clonal dominance") of metastatically competent tumor cell variants expressed under selective two- and three-dimensional tissue culture conditions. In vitro Cellular and Developmental Biology. Animal, 29A(9), 742-748.

[24] Siegel, G., Malmsten, M. and Klussendorf, D. (1998). Tumor cell locomotion and metastatic spread. Microscopy Research and Technique, 43, 276-282.

[25] Silletti, S., Paku, S. and Raz, A. (1998). Autocrine motility factor and the extracellular matrix. I. Coordinate regulation of melanoma cell adhesion, spreading and migration involves focal contact reorganization. International Journal of Cancer, $76,120-128$

[26] Solyanik, G.I., Bulkiewicz, R.I. and Kulik, G.I. (1995). One of the mechanisms of the metastatic cell dominance in heterogeneous tumour. Experimental Oncology, 17(2), 158.

[27] Solyanik, G.I. (1998). Mechanism of the metastatic cells overgrowth in a heterogeneous tumour. Physics of the Alive, $6(2), 59-64$.

[28] Staroselsky, A., Pathak, S. and Fidler, I.J. (1990). Changes in clonal composition during in vivo growth of mixed subpopulations derived from the murine K-1735 melanoma. Anticancer Research, 10, 291-296.

[29] Staroselsky, A.N., Radinsky, R., Filder, I.J., Pathak, S., Chernajovsky, Y. and Frost, P. (1992). The use of molecular genetic markers to demonstrate the effect of organ environment on clonal dominance in a human renal-cell carcinoma grown in nude mice. International Joumal of Cancer, 51(1), 130-138.

[30] Suh, O. and Weiss, L. (1984). The development of a technique for the morphometric analysis of invasion in cancer. Journal of Theoretical Biology, 107, 547-562.

[31] Symmans, W.F., Liu, J., Knowles, D.M. and Inghirami, G. (1995). Breast cancer heterogeneity: evaluation of clonality 
in primary and metastatic lesions. Human Pathology, 26(2), $210-216$.

[32] Takaoka, A., Hinoda, Y., Satoh, S., Adachi, Y., Itoh, F., Adachi, M. and Imai, K. (1998). Suppression of invasive properties of colon cancer cells by a metastasis suppressor KAIl gene. Oncogene, 16, 1443-1453.

[33] Theodorescu, D., Cornil, I., Sheehan, C., Man, S. and Kerbel, R.S. (1991). Dominance of metastatically competent cells in primary murine breast neoplasms is necessary for distant metastatic spread. International Journal of Cancer, 47, 118123.

[34] Traqui, P. (1995). From passive diffusion to active cellular migration in mathematical models of tumor invasion. Acta Biotheoretical, 43, 443-464.

[35] Uchida, S., Shimada, Y., Watanabe, G., Li, Z.G., Hong, T., Miyake, M. and Imamura, M. (1999). Motility-related protein (MRP-1/CD9) and KAI1/CD82 expression inversely correlate with lymph node metastasis in oesophageal squamous cell carcinoma. British Journal of Cancer, 79, 11681173.

[36] Uemura, K., Takao, S. and Aikou, T. (1998). In vitro determination of basement membrane invasion predicts liver metas- tases in human gastrointestinal carcinoma. Cancer Research, $58,3727-3731$.

[37] Volk, T., Geiger, B. and Raz, A. (1984) Motility and adhesive properties of high-and low-metastatic murine neoplastic cells. Cancer Research, 44(2), 811-824.

[38] Waghorne, C., Thomas, M., Lagarde, A., Kerbel, R.S. and Breitman, M.L. (1988). Genetic evidence for progressive selection and overgrowth of primary tumors by metastatic cell subpopulations. Cancer Research, 48, 6109-6114.

[39] Weiss, L. (1990). Metastatic inefficiency. Advances in cancer research, 54, 159-211.

[40] Zaizen, Y., Taniguchi, S. and Suita, S. (1998). The role of cellular motility in the invasion of human neuroblastoma cells with or without $\mathrm{N}$-myc amplification and expression. Journal of Pediatric Surgery, 33, 1765-1770.

[41] Zanker, K.S. (1997). To move or not to move, that is a question: two different paradigms of locomoting cells - the lymphocyte and the metastasic tumour cell. Cancer Letters, 14, $131-134$

[42] Ziober, B.l., Chen, Y.Q., Ramos, D.M., Waleh, N. and Kramer, R.H. (1999). Expression of the alpha7betal laminin receptor suppresses melanoma growth and metastatic potential. Cell Growth and Differentiation, 10, 479-490. 


\section{APPENDIX}

Here, we deduce equations into which the set (1) is transformed due to the condition (2), i.e. to the limitation of the total cell density.

Let us consider a multicellular system consisting of several intermixed subpopulations. Their total cell density is constant and equal to $A$ (for simplicity we assume that cells are of equal volumes; this approach is easily generalised on the case when cell volumes of different subpopulations are not the same). Let $a_{i}$ is the density of the $i$-th tumour cell population $(i \in(1$, $N)$ ), which can grow or transform into each other depending on the external conditions. Besides these tumour cell populations the system may include also "inert" or host cells (let's denote them by $b$ ), which are assumed to be immotile, do not grow but can be influenced by tumour cells (for instance killed by them due to lysis).

Evolution of the cell densities in some region $G \subset$ $R^{3}$ is in general case described by the following equations

$$
\begin{aligned}
& \dot{a}_{i}=f_{i}(\mathrm{a}, \cdot)-\nabla \cdot \mathrm{J}_{i}, \\
& \dot{b}=f_{b}(\mathrm{a}, \cdot)-\nabla \cdot \mathrm{J}_{b} .
\end{aligned}
$$

Here $f_{i}(\mathrm{a}, \cdot)$ are functions describing local kinetics of cell populations depending both on the cell densities and on the external conditions, for instance, on the concentration of nutrient $S$.

The variable $\mathbf{J}_{i}$ denotes the flux of the $i$-th population. It has two components: the flux determined by the own motility $\mathbf{J}_{i}{ }^{(1)}$, including in our case only random motility, described by the term $-D_{i} \nabla a_{i}$ (some motility coefficients $D_{i}$ may be equal to zero), and the convection flux $\mathbf{J}_{i}{ }^{(2)}=a_{i} \mathbf{V}$, where $\mathbf{V}$ is the velocity of the convective motion. The flux $\mathbf{J}_{b}$ consists of the convection component $b \mathbf{V}$ only.

Thus the set (8) takes the following appearance:

$$
\begin{aligned}
\dot{a}_{i} & =f_{i}(\mathrm{a}, S)+\nabla \cdot\left(D_{i} \nabla \mathrm{a}_{i}-a_{i} \mathrm{~V}\right), \\
\dot{b} & =f_{b}(\mathrm{a}, S)-\nabla \cdot(b \mathrm{~V}) .
\end{aligned}
$$

With the account of $\Sigma_{i} a_{i}+b=A$, summing the right- and left-hand sides of the equations (9), we obtain

$$
\nabla \cdot V=\frac{1}{A}\left(f_{b}(\mathrm{a}, S)+\sum_{j}\left(f_{j}(\mathrm{a}, S)+\nabla \cdot\left(D_{j} \nabla a_{j}\right)\right) .\right.
$$

After some manipulation, the details of which may be found in (Gusev and Polezhaev, 1997), in general case we arrive at a set of $\mathrm{N}+1$ equations: $\mathrm{N}$ parabolic equations for the cell densities and one elliptic (Poisson) equation for the scalar field, gradient of which determines the velocity field of the convective motion.

In the case of spherical symmetry considered in the present paper we may act in a different way as now $\mathbf{V}$ is in fact a scalar depending only on the radius and it is equal to zero in the origin. Then the equation (10) can be solved directly which gives

$$
\mathrm{V}=\frac{1}{A}\left(\frac{1}{r^{2}} \int_{0}^{r} \rho^{2} F(\mathrm{a}, S) d \rho+\sum_{j}\left(D_{j} \nabla a_{j}\right)\right),
$$

where $F$ is determined by the expression

$$
F(\mathrm{a}, S) \equiv f_{b}(\mathrm{a}, S)+\sum_{j} f_{j}(\mathrm{a}, S) .
$$

Substituting (12) into the set (9) we obtain

$$
\begin{aligned}
\dot{a}_{i}= & \sum_{j}\left[\left(\delta_{i j}-\frac{a_{i}}{A}\right) f_{j}(\mathrm{a}, \cdot)\right. \\
& \left.+\frac{1}{r^{2}} \frac{\partial}{\partial r}\left(D_{j} r^{2}\left(\delta_{i j}-\frac{a_{i}}{A}\right) \frac{\partial a_{i}}{\partial r}\right)\right] \\
& -\frac{1}{A} \frac{\partial a_{i}}{\partial r} \cdot \frac{1}{r^{2}} \int_{0}^{r} \rho^{2} F(\mathrm{a}, S) d \rho .
\end{aligned}
$$

An important question concerns the specific form of the function $f_{b}$ describing local kinetics of the host cells.

It is known that some types of tumours in the process of their growth kill host tissue cells by lysis, others only push them away clearing space for the offspring. In most cases both processes occur, the difference is in the degree of their realisation. As is mentioned above a high rate of the proteolytic cascade in metastatically competent cells is usually observed. Thus in the present research we consider an extreme case and assume that tumour cells do not experience 
any resistance from the host tissues, the former do not push the latter but only kill them by lysis.

The local kinetics of the host cells may formally be described in this case by the following function

$$
\begin{aligned}
f_{b}(\mathrm{a}, S)= & -\theta\left(\sum_{i} a_{i}-A\right) \sum_{j} f_{j}(\mathrm{a}, S) \\
& -\delta\left(\sum_{i} a_{i}-A\right) \nabla \cdot(A \mathrm{~V}),
\end{aligned}
$$

where $\delta$ is a simbolic Dirac delta-function. This form of the function $f_{b}(\mathbf{a}, S)$ ensures both (i) zero value of the function $F(\mathbf{a}, S)$ which is a source term for the vector field $\mathbf{V}$ (see (11)) and (ii) zero value of the convective flow $\mathbf{V}$ outside the tumor.

If we substitute (14) into (12) we will see that outside the region of tightly packed tumour cells where the host tissue cells are present, the equations (13) are reduced to the form of conventional reaction-diffusion equations and thus cells can move due to their locomotion capacity only. Meanwhile inside the region void of host tissue tumour cells achieve maximal possible total density and move due to both their own random motility and convective motion. 


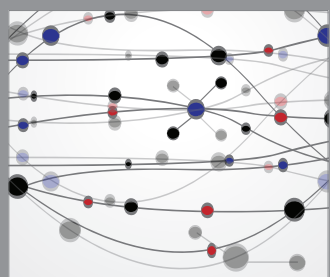

The Scientific World Journal
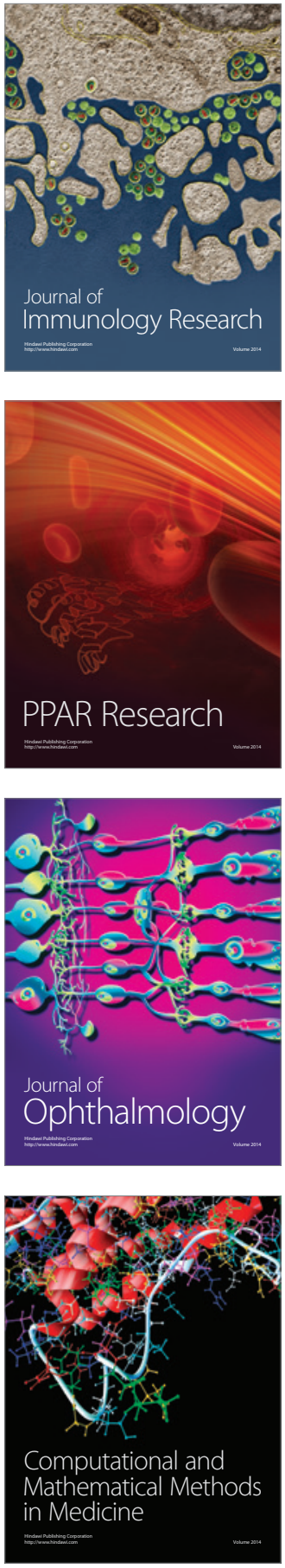

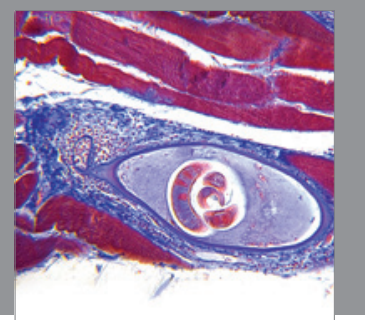

Gastroenterology

Research and Practice
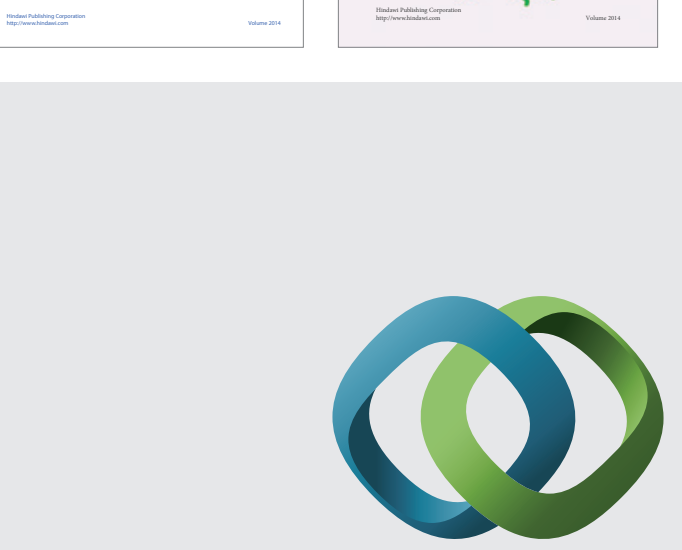

\section{Hindawi}

Submit your manuscripts at

http://www.hindawi.com
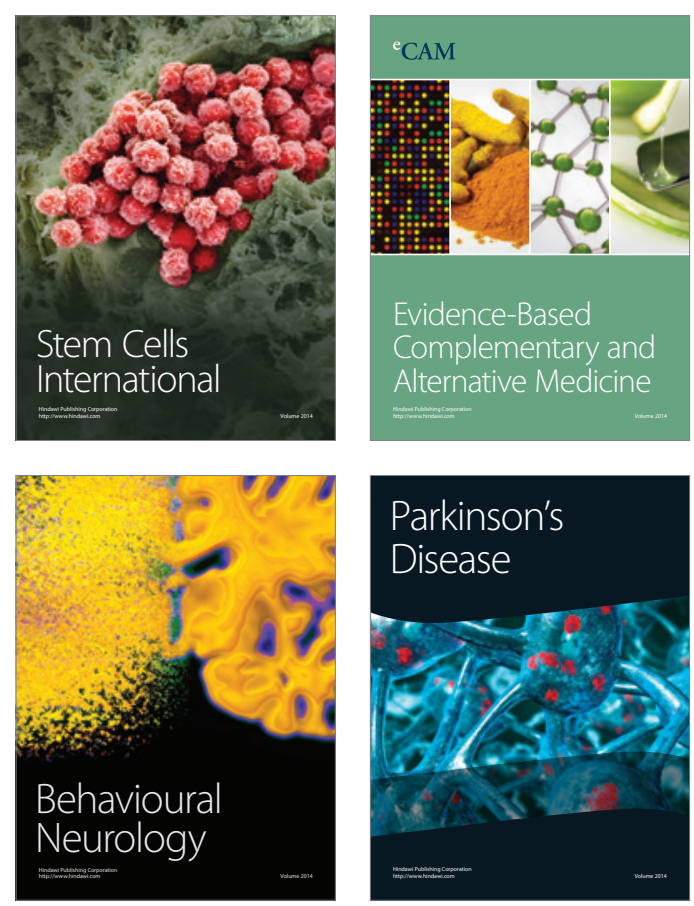

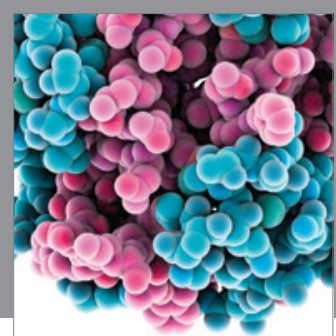

Journal of
Diabetes Research

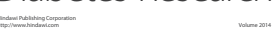

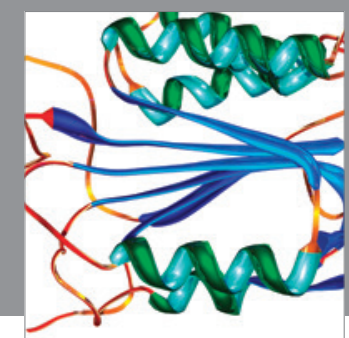

Disease Markers
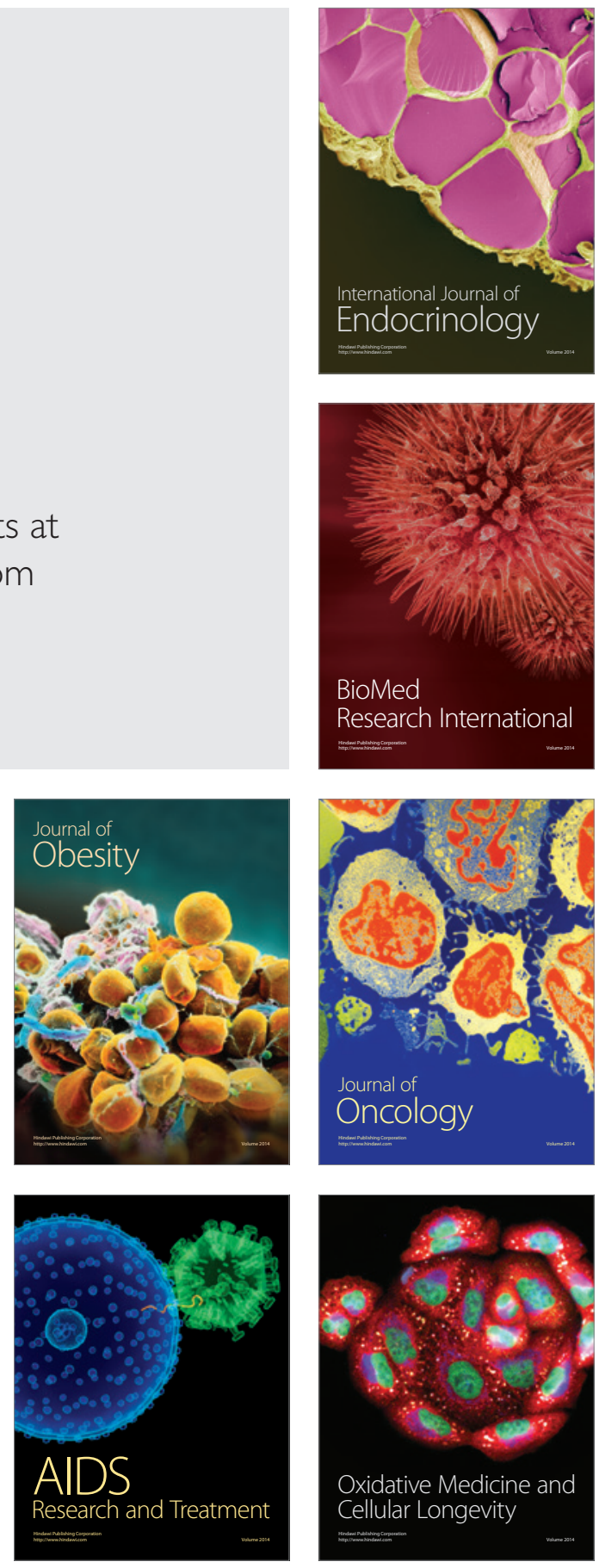